\title{
Observer Variation in the Clinical and Radiological Assessment of Hepatosplenomegaly
}

\author{
L. M. BLENDIS, ${ }^{*}$ M.B., M.R.C.P. ; W. J. McNEILLY, $\dagger$ M.R.C.P., D.M.R.D. ; LOUISE SHEPPARD, $\dagger$ M.B., D.M.R.D. \\ ROGER WILLIAMS, $\ddagger$ M.D., F.R.C.P. ; J. W. LAWS, $\oint$ F.R.C.P., F.F.R.
}

ummary: The size of the liver and spleen of 32 patients $\sim$ was assessed by four clinicians from a clinical examination and by four radiologists from a plain radiograph of the abdomen. The latter assessment was found to be subject to less variation than the former, particularly in regard to the liver. Large livers and spleens were more easily seen radiologically than small ones. Most of the variation among the radiologists arose because of the $6 \%$ and $24 \%$ of cases in which the liver and spleen, respectively, were poorly seen on the radiograph. It is concluded that a plain radiograph of the abdomen, including the diaphragm and with the costal margin indicated, is a useful adjunct to clinical examination.

\section{Introduction}

A combination of palpation and percussion of the upper abdomen is regarded by most clinicians as satisfactory for diagnosing enlargement of the liver and spleen. Yet these physical signs have not been critically analyzed for observer error and variation in the same way as those, for example, of the respiratory system (Fletcher, 1952; Smyllie, Blendis, and Armitage, 1965; Godfrey, Edwards, Campbell, Armitage, and Oppenheimer, 1969). Another common way of assessing liver and spleen size is from a plain abdominal radiograph, but again this method has not been assessed critically in the same way as chest radiographs (Cochrane and Garland, 1952).

In this paper we describe an investigation in which the variation between four clinicians in examining the liver and spleen was compared with that found when four radiologists assessed the size of the liver and spleen on plain films of the abdomen.

\section{Patients Studied and Method}

Thirty-two patients were examined. The purpose of the investigation had previously been explained to the patients and their full co-operation obtained. Fourteen had a blood dyscrasia (three havin ? : ated by splenectomy), 10 had chronic liver disease, nd 8 suffering from a variety of other conditions.

Each patient was sc

ur clinicians on the same afternoon and a form was a the measurements of liver and spleen size (Fig. 1) were n ade on quiet expiration.

A plain film of the abdomen was taken on quiet expiration with the patient in the supine position at an anode-film distance of $120 \mathrm{~cm}$. and centred at the xiphisternum. Both domes of the diaphragm and both lateral abdominal walls were included on the radiograph and the position of the costal margin and sterno-xiphisternal junction was marked on the radio-

\footnotetext{
* Research Fellow, Medical Research Council Group on Metabolism and Haemodynamics of Liver Disease, Department of Medicine. $\dagger$ Registrar, Department of Diagnostic Radiology.

‡ Director, Medical Research Council Group on Metabolism and Haemodynamics of Liver Disease, Department of Medicine. Director, Department of Diagnostic Radiology, King's College Hos-
pital, London S.E.5.
}

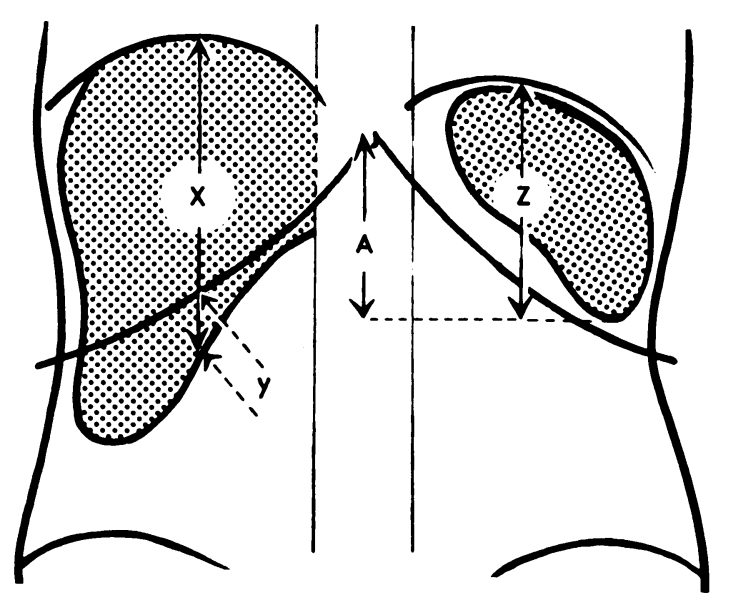

FIG. 1.-Various measurements made by the clinicians and radiologists.

graph by outlining the surface markings with a thin radioopaque wire. The height and weight of each patient was recorded at the time of examination, and their build as obese, normal, or thin.

The radiographs were assessed and measurements made independently by four radiologists are corrected for magnification. The radiologists made the same linear measurements as the clinicians (Fig. 1). The area of the liver to the right of the spine and the complete area of the spleen were also measured with a planimeter. These measurements were then corrected for body surface area and expressed in sq. $\mathrm{cm}$. per sq. $\mathrm{m}$. body surface area. Each radiograph was graded independently by the four radiologists according to the clarity of the hepatic and splenic outline into good, fair, poor, and impossible. The visibility of the tip of the spleen was graded separately. In addition the liver area was measured from a plain film of the abdomen in 44 patients attending the department of diagnostic radiology for intravenous pyelography as part of the investigation of systemic hypertension or renal disease. These formed a control group.

The observer variation was assessed as the difference between the largest and smallest values recorded by the observers. This was called the "maximum observer variation." In order to relate this to the size of the measurement it was also expressed as a percentage of the mean of the four measurements, the "percentage maximum observer variation."

Results
Liver
Good agreement was found between the four radiologists in
grading the ease with which the liver to the right of the spine
could be seen on the radiographs. At least three radiologists
agreed on the grading in $94 \%$ of the radiographs and all
agreed in $70 \%$. Visibility was graded by the majority as good
in $55 \%$, fair in $33 \%$, and poor in $6 \%$ of patients. The ease


with which the liver was seen was independent of the patient's body build, but large livers were seen more easily than small (Table I).

TABLE I.-Area of Liver and Ease with which Outline was Seen on Plain Radiograph

\begin{tabular}{|c|c|c|c|}
\hline \multirow{2}{*}{$\begin{array}{c}\text { Liver Area } \\
\text { (sq. cm./sq. m.) }\end{array}$} & \multicolumn{3}{|c|}{ Percentage of Livers in Each Group } \\
\hline & Good & Fair & Poor \\
\hline $\begin{array}{c}68-82 \\
83-109 \ldots \\
>109 \quad \ldots\end{array}$ & $\begin{array}{l}20 \\
54 \\
87\end{array}$ & $\begin{array}{l}60 \\
38 \\
13\end{array}$ & $\begin{array}{r}20 \\
8 \\
0\end{array}$ \\
\hline
\end{tabular}

Liver Area.-The liver area ranged from 68 to 152 sq. $\mathrm{cm}$./sq. m., the mean of the four radiologists' measurements being used. The maximum variation between these observers ranged from 0 to $22 \mathrm{sq}$. $\mathrm{cm}$./sq. m. The percentage maximum observer variation averaged $9 \%$, with a range of $0-21 \%$, and the effect of visibility on this variation is shown in Table II. These values for liver area are to be compared with the mean value of $82 \mathrm{sq} . \mathrm{cm}$./sq. m. (S.D. 13.4) in the control group determined by two observers.

TABLE II.-Observer Variation and Ease with which Liver was Seen on Plain Radiograph

\begin{tabular}{|c|c|c|c|c|}
\hline \multirow{3}{*}{ Visibility } & \multicolumn{4}{|c|}{ Liver Area } \\
\hline & \multicolumn{2}{|c|}{$\begin{array}{c}\text { Maximum } \\
\text { Observer Variation (sq. cm.) }\end{array}$} & \multicolumn{2}{|c|}{$\begin{array}{c}\% \text { Maximum } \\
\text { Observer Variation }\end{array}$} \\
\hline & Mean & Range & Mean & Range \\
\hline $\begin{array}{l}\text { Good .. } \\
\text { Fair } \quad \ldots \\
\text { Poor } \quad .\end{array}$ & $\begin{array}{r}6 \\
9 \\
21\end{array}$ & $\begin{array}{r}0-18 \\
5-13 \\
19-22\end{array}$ & $\begin{array}{r}6 \\
10 \\
17\end{array}$ & $\begin{array}{r}0-16 \\
4-21 \\
15-20\end{array}$ \\
\hline
\end{tabular}

Clinical Assessment of Liver Enlargement.-The variation of each clinician's measurements from the mean of the four measurements on each patient was analysed and found not to differ significantly. This excludes the possibility of one inaccurate observer consistently increasing the maximum observer variation. An assessment of liver enlargement was recorded by all four clinicians in 28 of the 32 patients in this study. There was agreement in their assessment of whether the liver was enlarged or not in 15 patients $(54 \%)$ and in a further 11 three of the four observers agreed. Most of the observers agreed on whether the liver was enlarged in $93 \%$ of patients. The effect of body build and liver area on the variation between the observers' assessment was analysed, but no significant difference was found. Clinical assessment of enlargement of the liver was compared with the radiological area in 25 of the patients in whom at least three clinicians agreed in their assessment of size. In six patients the liver area was greater than 109 sq. $\mathrm{cm}$. (the upper limit of the normal range obtained from the control group, using the mean value plus two standard deviations) and the liver was clinically assessed as enlarged in three and normal in three. In the other 19 patients the liver area was within the normal range and the liver was clinically assessed as enlarged in 10 and normal in nine.

Liver Length.-The vertical length from the upper to the lower border of the liver in the mid-clavicular line when measured on the radiograph ranged from 9.2 to $23.5 \mathrm{~cm}$. when the mean of the four radiologists' readings was taken. The mean maximum variation between the four radiologists was $1.3 \mathrm{~cm}$., or $8 \%$ (range $0-52 \%$ ). In comparison the mean of the four clinicians' measurements ranged from 10.5 to $26 \mathrm{~cm}$. The mean maximum variation between the four clinicians was $8 \mathrm{~cm}$., or $55 \%$ (range $0-100 \%$ ). Thus, in one patient all four clinicians agreed exactly, and in another the maximum variation was as large as the mean of the four measurements. There was no significant difference in the variation between observers when the patients were grouped according to body build. The clinical and radiological measurements of the distance of the lower border of the liver below the costal margin in the mid-clavicular line are shown in Fig. 2. The maximum

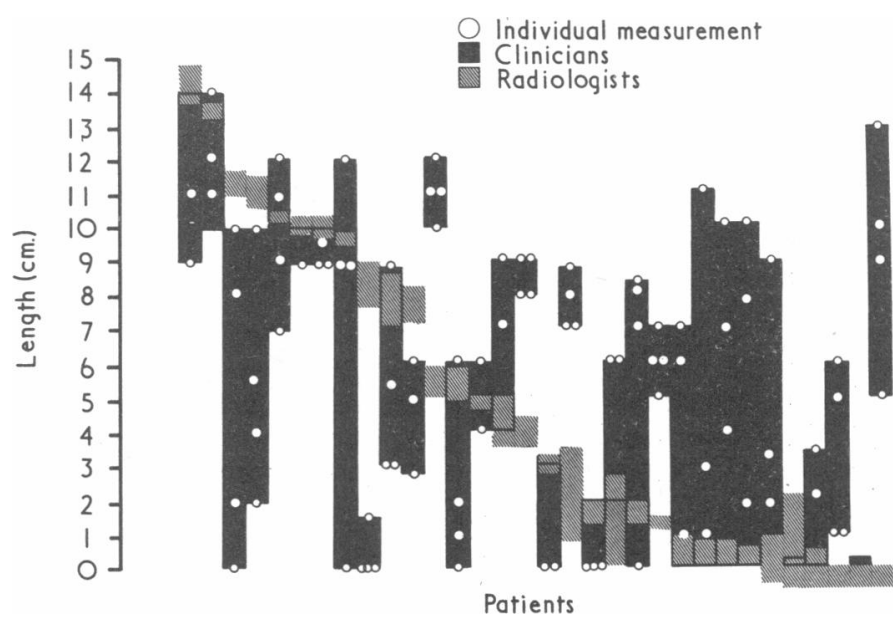

FIG. 2.- Individual and range of measurements of the clinicians for the distance of the lower border compared with the range for the same measurements made by the radiologists on the radiograph.

variation between the recordings of the four radiologists was small, the mean being $1 \mathrm{~cm}$. (range 0 to $3 \mathrm{~cm}$.). In comparison the mean maximum variation between the four clinicians was $5 \mathrm{~cm}$. (range 0 to $12 \mathrm{~cm}$.).

The relation between the radiological distance below the costal margin, liver area, and clinical assessment of liver size was also studied. In five patients the liver did not extend below the costal margin, and the areas of these livers were all in the lower half of the normal range. In the six patients with liver areas greater than normal the liver extended from 5 to 15 $\mathrm{cm}$. below the costal margin. In five of the patients with normal liver areas, however, the liver extended from 5 to $10 \mathrm{~cm}$. below the costal margin. In all five of these patients the diaphragm was not abnormally low as assessed clinically by percussion or on the radiographs, but the liver area in four of them was near the upper limit of the normal range.

\section{Spleen}

All four radiologists agreed on the clarity of the outline of the spleen in $54 \%$ of radiographs and at least three agreed in $90 \%$. Similarly, all four radiologists agreed on the visibility of the spleen tip in $65 \%$ and at least three agreed in $87 \%$. This finding included six patients in whom all four radiologists agreed that it was impossible to see the spleen. Three of these patients, unknown to the radiologists, had undergone splenectomy, and in the other three the spleen was clinically impalpable. In the 29 patients with a spleen the clarity of the splenic outline was graded by most observers as good in $41 \%$, fair in $21 \%$, and poor in $24 \%$. Corresponding figures for the spleen tip were $54 \%, 20 \%$, and $14 \%$ respectively. When the patients were divided into three groups according to the spleen area it was found that large spleens were more easily seen than small (Tables III and IV).

The spleen area ranged from 34 to 238 sq. cm./sq. m., the mean of the radiologists' measurements being used. This is to be compared with a normal range of 40 (S.D. 4) sq. cm./sq. m. derived by Blendis, Williams, and Kreel (1969). The mean maximum variation between the four radiologists was $15 \mathrm{sq} . \mathrm{cm}$., or $22 \%$ (range $1-94 \%$ ). The effect of radiographic visibility is shown in Table V.

The distance of the spleen tip below the xiphisternalsternal junction ranged from 2 to $22 \mathrm{~cm}$. The mean maximum observer variation was $2 \mathrm{~cm}$. or $19 \%$ (range $0-126 \%$ ). The effect of radiographic visibility is shown in Table $\mathrm{V}$. 
TABLE III.-Area of Spleen and Ease with which Outline was Seen on Plain Radiograph

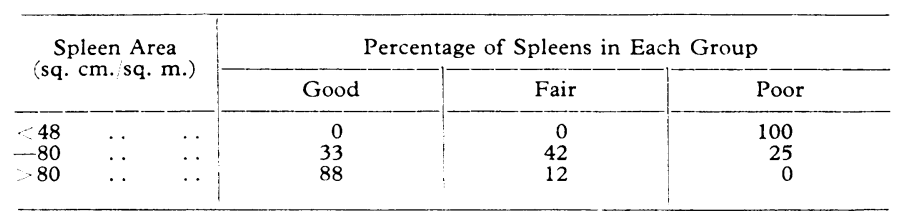

TABLE IV.-Area of Spleen and Ease with which Lower Pole was Seen on Plain Radiograph

\begin{tabular}{|c|c|c|c|}
\hline \multirow{2}{*}{$\begin{array}{c}\text { Spleen Area } \\
(\text { sq. cm./sq. m.) }\end{array}$} & \multicolumn{3}{|c|}{ Percentage of Spleens in Each Group } \\
\hline & Good & Fair & Poor \\
\hline $\begin{array}{lll}<48 & \ldots & \ldots \\
48-80 & \ldots & \ldots \\
>80 & \ldots & \ldots\end{array}$ & $\begin{array}{r}0 \\
59 \\
88\end{array}$ & $\begin{array}{l}25 \\
33 \\
12\end{array}$ & $\begin{array}{r}75 \\
8 \\
0\end{array}$ \\
\hline
\end{tabular}

TABLE V.-Observer Variation and Ease with which Spleen was Seen on Plain Radiograph

\begin{tabular}{|c|c|c|c|c|}
\hline \multirow[t]{2}{*}{ Visibility } & \multicolumn{2}{|c|}{$\begin{array}{l}\text { Spleen Area } \\
\text { Maximum Observer Variation } \\
(\text { sq. cm.) }\end{array}$} & \multicolumn{2}{|c|}{$\begin{array}{l}\text { Spleen Length } \\
\text { Maximum Observer Variation } \\
\text { (cm.) }\end{array}$} \\
\hline & Mean & Range & Mean & Range \\
\hline $\begin{array}{l}\text { Good } \ldots \\
\text { Fair } \\
\text { Poor }\end{array}$ & $\begin{array}{l}13(10 \%) \\
11(16 \%) \\
27(65 \%)\end{array}$ & $\begin{array}{l}1-63(1-26 \%) \\
4-31(4-40 \%) \\
6-35(14-94 \%)\end{array}$ & $\begin{array}{l}1 \quad(7 \%) \\
3(21 \%) \\
2(62 \%)\end{array}$ & $\begin{array}{l}0-2(0-23 \%) \\
1-7(3-40 \%) \\
1-4(10-126 \%)\end{array}$ \\
\hline
\end{tabular}

Clinical Assessment.-All four observers agreed on an assessment of whether the spleen was enlarged or not in 28 of the 32 patients $(88 \%)$, and in 31 patients $(97 \%)$ three of the four observers agreed. The assessment was not significantly affected by spleen size or body build. In all 15 patients in whom at least three clinicians diagnosed splenic enlargement the spleen area expressed as a mean of the four radiologists' measurements was greater than $48 \mathrm{sq} . \mathrm{cm}$./sq. m., which is the upper limit of the normal range of splenic area (Blendis et al., 1969). In 8 of the 17 patients diagnosed clinically as having normal-sized spleens the spleen area could be measured radiologically. In four the spleen areas were within the normal range. In two patients the spleen areas were increased (66 and 69 sq. cm./sq. m.). In the remaining two patients the spleen area was 50 sq. cm./sq. m., just above the upper limit of normal.

All four clinicians were able to palpate the spleen and therefore measure the distance of the spleen tip below the ziphisternal-sternal junction. in 12 patients. Their mean maximum observer variation was $6 \mathrm{~cm}$. compared with $2 \mathrm{~cm}$. for the radiologists in these 12 patients.

\section{Discussion}

In any assessment of a radiological measurement, such as that of liver and spleen size, the question of the ease with which the landmarks can be seen must be considered. In the present series there was agreement between the majority of radiologists in over $80 \%$ of the radiographs. Though visibility was not related to body build it was related to the size of the organ. Thus all the large livers and most of those in the upper half of the normal range were better seen on the radiographs than were the smaller ones. The visibility of enlarged spleens was satisfactory, but that of normal-sized spleens was not. These findings agree with those of Rosenbaum, Lieber, Hanson, and Pellegrino (1964), who were able to see only $17 \%$ of the spleens on 500 consecutive abdominal radiographs. Riemenschneider and Whalen (1965), however, found that 16 out of 30 spleens under $200 \mathrm{~g}$. weight were adequately seen, though this probably refers only to the lower pole.

Though the lower pole of the spleen was more clearly seen than the rest of the outline, confirming findings in previous series (Wyman, 1954), the extent of the variation between observers in the measurements of the area and length of the spleen taken from the radiographs was of the same order.
A potential source of inaccuracy in the measurement of splenic area in a plain radiograph is the assumption, which it is often necessary to make, that the upper and lateral borders of the spleen lie immediately adjacent to the diaphragm and lateral abdominal wall. Arteriographic studies show that this is not always correct (Kreel and Mindel, 1969). In eight patients it was possible to compare the measurement of splenic area from the plain film with that made from a splenic arteriogram done within four weeks, and the mean difference was only $8 \%$.

The variation between observers in the linear measurements of the liver and spleen was much less for radiologists, than for clinicians. The observer variation of the radiologists, as migh be expected, decreased with better visibility on the radiographs and was not significantly affected by body build of the patients. Surprisingly, the variation between the clinicians was not significantly affected by body build or by organ size.

The criteria for the clinical diagnosis of hepatic enlargement have not been cleary defined. If the lower border of the liver is palpable more than $2-3 \mathrm{~cm}$. below the costal margin the liver is usually considered to be enlarged. This study, however, has shown a wide observer variation for this measurement. Whalen, Berne, and Riemenschneider (1969) suggested that the lower border of the liver, as seen on a radiograph, is in fact the posterior border. To determine whether this is so radio-opaque markers were placed on the skin of seven cadavers and radiographs taken of the upper abdomen. A long needle was inserted into the cadaver at the lower border of the liver as estimated from the radiograph. In each case, at the subsequent necropsy, the needle was found at or just below the anterior lower border of the liver.

The variation between clinicians in the measurement of the distance of the lower pole of the spleen below the xiphisternal-sternal junction was less than for the liver length. This may be due to the former having one fixed, easily determined point. There was also nearly complete agreement among the clinicians in diagnosing splenic enlargement. Though Dell and Klinefelter (1946) suggested that normal spleens might be palpable, this was not confirmed in necropsy study by Riemenschneider and Whalen (1965). The weight of the spleen has been shown to correlate closely with radiographic area in both normal and enlarged spleens and to bear a fairly constant relationship to body surface area (Blendis et al., 1969). In the present series all 15 of the spleens which were palpable were enlarged as judged by radiological area. These included five spleens with 25 to $50 \%$ increase in radiological area. This agrees with the findings of Blackburn (1953), who was able to detect clinically a $40 \%$ increase in spleen size as estimated radiographically. Occasionally, however, as shown in the present series, even a considerably enlarged spleen can be missed on palpation.

This is in keeping with the findings of McCready and Ahuja (1969) and McCready, Gwyther, and Stringer (1969). who found that only 34 out of 76 patients with Hodgkin's disease had spleens that were palpable, though 43 were enlarged as estimated by rectilinear scanning after the injection of labelled heat-denatured autologous red blood cells. They conclude that the best diagnostic result is obtained when the scan is examined in conjunction with scans of other surrounding organs and the appropriate radiographs. Estimation of spleen size by means of a gamma camera scintigram gives a less accurate result.

In conclusion, this study suggests that in the diagnosis of hepatosplenomegaly, particularly with reference to enlargement of the liver, a plain film of the abdomen with the costal margin indicated is subject to less observer variation than clinical examination and also allows a quantitative assessment of the size of these organs.

We thank Dr. R. P. H. Thompson, Dr. I. Murray-Lyon and Dr. R. Wilkins for taking part in the clinical and radiological studies, and Miss Berry and the radiographic staff, who gave invaluable assistance. 


\section{REFERENCES}

Blackburn, C. R. B. (1953). Australasian Annals of Medicine, 2, 78.

Blendis, L. M., Williams, R., and Kreel, L (1969). Gut, 10, 433.

Cochrane, A. L., and Garland, L. H. (1952). Lancet, 2, 505 .

Dell, J. M., jun., and Klinefelter, H. F. (1946). American fournal of Medical Sciences, $211,437$.

Fletcher, C. M. (1952). Proceedings of the Royal Society of Medicine, 45, 577 .

Godfrey, S., Edwards, R. H. T., Campbell, E. J. M., Armitage, P., and Oppenheimer, E. A. (1969). Thorax, 24, 4

Kreel, L., and Mindel, S. (1969). British fournal of Radiology, 42, 830. McCready, V. R., and Ahuja, S. (1969). Proceedings of the Royal Society of Medicine, 62, 794.
McCready, V. R., Gwyther, M. M., and Stringer, A. (1969). In Medical Radioisotope Scintigraphy, vol. 2, p. 419. Vienna, International Atomic Energy Agency.

Riemenschneider, P. A., and Whalen, J. P. (1965). American fournal of Roentgenology, Radium Therapy and Nuclear Medicine, 94, 462.

Rosenbaum, H. D., Lieber, A., Hanson, D. J., and Pellegrino, E. D. (1964). American fournal of Roentgenology, Radium Therapy and Nuclear Medicine, 91, 903.

Smyllie, H. C., Blendis, L. M., and Armitage, P. (1965). Lancet, 2,

Whalen, J. P., Berne, A. S., and Riemenschneider, P. A. (1969), Radio$\log y, 92.466$.

Wyman, A. C. (1954). American fournal of Roentgenology, Radium Therapy and Nuclear Medicine, 72, 51

\title{
Erythropoietic Protoporphyria:* IV. Protection from Sunlight
}

\author{
RAMON M. FUSARO, $†$ M.D., PH.D. ; WALTER J. RUNGE, $\ddagger$ M.D., PH.D.
}

Summary: Dihydroxyacetone and lawsone, in a vanish$N$ ing cream base, applied to the skin was found to protect patients with erythropoietic protoporphyria against sunlight. The use of the same ingredients in a $50 \%$ isopropyl alcohol/water solution did not induce adequate light protection. The chemically induced ultraviolet light filter in the stratum corneum allowed these patients to change their lives from an "indoor" to an "outdoor" environment.

\section{Introduction}

Several years ago we introduced a new concept of protection against ultraviolet light (Runge and Fusaro, 1962; Fusaro and Runge, 1962). That concept consists of chemically altering the stratum corneum so that the keratin has new ultraviolet transmission characteristics. The new keratin is produced by chemically reacting a dihydroxyacetone/naphthoquinone mixture with the skin at least 24 hours before exposure to sunlight. This altered keratin reduces significantly the quanta of ultraviolet light penetrating the skin, thereby redusing the possibility of a photodynamic effect of ultraviolet light on the porphyrin molecule in the skin. We here deal with the application of this concept for the protection of erythropoietic protoporphyric patients against sunlight.

\section{Materials and Methods}

The patients were given a topical mixture of $3 \%$ dihydroxyacetone and $0.13 \%$ lawsone in a chemically compatible vanishing-cream base (Squibb Experimental Base). The cream was made by adding the dihydroxyacetone powder direct to the cream and adding the lawsone dissolved in a small quantity of isopropyl alcohol $(5 \mathrm{mk} . / \mathrm{ml}$.). The active principles were also used in a $50 \%$ solution of isopropyl alcohol and distilled water. If the solution was used it was dispensed as two solutions in order to assure stability; $6 \%$ dihydroxyacetone in $50 \%$ solution of isopropyl alcohol and distilled water and $0.25 \%$ lawsone in $50 \%$ solution of isopropyl alcohol and distilled water. The day the patient was to be treated he mixed equal parts of the two solutions and sprayed (aerosol spray) the final mixture on the skin.

\footnotetext{
* The value of normal erythrocyte protoporphyrin is $15-65 \mu \mathrm{g} . / 100 \mathrm{ml}$. of blood and not the printer's error of $565 \mu \mathrm{g}$. as seen in our first article (Peterka et al., 1965).

† Associate Professor, Department of Dermatology, University of Minnesota Medical School, Minneapolis, Minnesota, U.S.A.

¥ Assistant Professor, Department of Pathology, University of Minnesota Medical School, Minneapolis, Minnesota, U.S.A.
}

The patients used the topical therapy after their cutaneous eruption had cleared. Any eruption before the protective treatment was given was treated with topical steroid therapy and by avoiding sufficient light exposure to cause symptoms. Steroid therapy was discontinued before the protective regimen was started. This precaution of clearing the eruption was necessary as an effective ultraviolet light filter can be induced only into an intact stratum corneum. The topical preparations were applied six to eight times a day for the first 48 hours. Thereafter each patient applied the preparations three times a day for the next five days. After the first week he was allowed to go out in the sunlight for what he considered "a prolonged period of time." In his past experience sunlight exposure for this period of time was usually followed by an outbreak of cutaneous symptoms or eruption. After the first exposure the patient applied the topical therapy one to four times a day, depending on his degree of light sensitivity. If the protection was judged inadequate because of excessive light exposure the therapeutic regimen was reinstituted; but in addition the number of topical applications was increased.

The patient was instructed not to wash his skin within one hour after the application. In some patients all the applications were applied late in the day (after 18.00 hours) with at least one hour between the applications. When the patient bathed he used only soap to clean his skin and not a "washcloth" or "scrub-brush." When drying his skin after a bath, he was to "pat his skin dry" and not "vigorously rub or towel down."

In attempting to set up criteria for judging degree of protection by the amount of sunlight the patient could tolerate it was apparent that the measure of time should be simple, be realistic, and reflect his clinical experience. An endpoint, determined by the patient, of the minimal amount of sun exposure needed to produce symptoms seemed to be the simplest measure of the efficiency of protection. The patient invariably remembered that experience in which he had the least protection. That clinical exposure usually represented the most drastic exposure to sunlight. An estimation of an average of the minimum did not appreciably clarify the situation as the following variables had to be considered in determining the average: (1) day of the year; (2) time of day in which exposure was made; (3) the condition of the surrounding environment-that is, buildings, trees, etc.; (4) weather conditionsthat is, type and percentage of cloud cover; (5) the amount of clothes-that is, hat, long or short sleeves, type of material-and the length of time each item was worn during the exposure -for example, was the hat worn all the time?; and (6) each patient's disease had a different degree of photosensitivity, depending on the severity of the chemical abnormality. It was apparent that an integration of these variables into a mean- 